\section{IN BRIEF}

\section{$\Rightarrow$ RHEUMATOID ARTHRITIS}

\section{Daily atorvastatin safe for patients with RA}

Data from the TRACE RA trial ( $n=3,002$; median follow-up 2.5 years) suggest that atorvastatin $40 \mathrm{mg}$ daily is safe and reduces $\mathrm{LDL}$ cholesterol in patients with rheumatoid arthritis (RA), but whether the statin therapy prevents major cardiovascular events (CVEs) remains unclear as the trial was terminated early owing to an unexpectedly low rate of CVEs. At the end of the study, mean LDL-cholesterol concentrations were $0.77 \mathrm{mmol} / \mathrm{l}$ lower in patients allocated to receive atorvastatin than in those who received placebo $(P<0.0001)$. $24(1.6 \%)$ patients in the atorvastatin group experienced a major CVE, compared with $36(2.4 \%)$ in the placebo group (HR 0.66; 95\% Cl, 0.39-1.11, $P=0.115$ ).

ORIGINAL ARTICLE Kitas, G. D. et al. Trial of atorvastatin for the primary prevention of cardiovascular events in patients with rheumatoid arthritis (TRACE RA): a multicenter, randomized, placebo controlled trial. Arthritis Rheumatol. https://doi.org/10.1002/art.40892 (2019)

\section{$\Rightarrow$ SYSTEMIC LUPUS ERYTHEMATOSUS}

\section{TIGIT-Ig shows therapeutic potential in SLE}

The immunomodulatory molecule and co-inhibitory receptor T cell immunoreceptor with Ig and ITIM domains (TIGIT) could be used to treat autoimmune diseases, according to results in a mouse model of lupus. Treatment of NZB/W F1 mice with a TIGIT-Ig fusion protein inhibited production of anti-doublestranded-DNA antibodies, delayed the development of proteinuria and prolonged survival in comparison with mice treated with control lgG. The TIGIT-Ig fusion protein demonstrated lgG-like stability similar to that of CTLA-4-lg. ORIGINAL ARTICLE Liu, S. et al. Treatment of murine lupus with TIGIT-Ig. Clin. Immunol. https://doi.org/10.1016/j.clim.2019.04.007 (2019)

\section{$\Rightarrow$ RHEUMATOID ARTHRITIS}

\section{Namilumab improves RA symptoms}

Treatment with an investigational antibody targeting granulocyte-macrophage colony-stimulating factor (GM-CSF) improved the signs and symptoms of rheumatoid arthritis (RA) in a dose-finding phase II randomized controlled trial. Patients with RA and an inadequate response to methotrexate $(n=92)$ or anti-TNF therapy $(n=16)$ received subcutaneous namilumab $(20 \mathrm{mg}, 80 \mathrm{mg}$ or $150 \mathrm{mg}$ ) or placebo at baseline and at weeks 2 . 6 and 10 . At week 12, disease activity was significantly reduced from baseline in patients treated with all doses of namilumab versus placebo $(P=0.005)$, with a dose-response effect apparent from week 2.

ORIGINAL ARTICLE Taylor, P. C. et al. Efficacy and safety of namilumab, a human monoclonal antibody against granulocyte-macrophage colony-stimulating factor (GM-CSF) ligand in patients with rheumatoid arthritis (RA) with either an inadequate response to background methotrexate therapy or an inadequate response or intolerance to an anti-TNF (tumour necrosis factor) biologic therapy: a randomized, controlled trial. Arthritis Res. Ther. 21, 101 (2019)

\section{$\Rightarrow$ EPIDEMIOLOGY}

\section{Burden of musculoskeletal diseases increasing}

The global burden of musculoskeletal diseases increased significantly between the years 2000 and 2015, as measured by the disability-adjusted life years index $(P<0.001)$, and is higher in Europe than in all other continents. Over the same period, musculoskeletal diseases remained the second leading cause of years lived with disability worldwide.

ORIGINAL ARTICLE Sebbag, E. et al. The world-wide burden of musculoskeletal diseases: a systematic analysis of the World Health Organization Burden of Diseases Database. Ann. Rheum. Dis. https://doi.org/10.1136/annrheumdis-2019-215142 (2019)
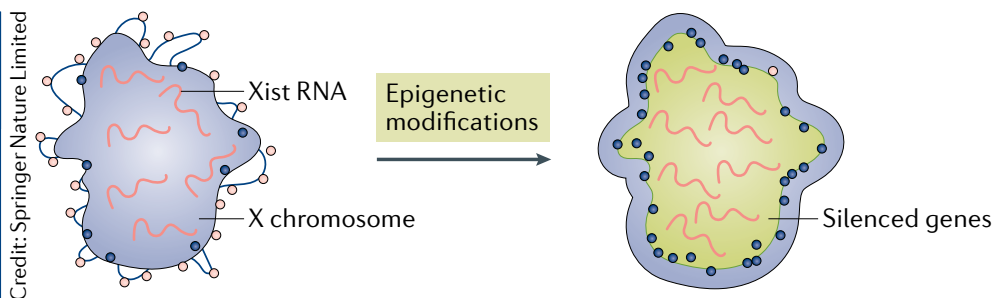

EPIGENETICS

\title{
X-chromosome inactivation altered in SLE
}

Sex bias in autoimmune diseases such as systemic lupus erythematosus (SLE) has been attributed to several factors, including sex hormones, sex chromosome gene dosage and microchimerism. A study published in JCI Insight provides new evidence for abnormal X-chromosome inactivation in $\mathrm{T}$ cells from patients with SLE, suggesting that faulty epigenetic control of gene dosage could be a factor contributing to sex bias in this disease.

In every cell containing two X chromosomes, one $\mathrm{X}$ chromosome is randomly chosen to be inactivated in a process orchestrated by the long noncoding RNA Xist, which recruits chromatin complexes that create epigenetic modifications, thereby silencing genes. "We were motivated to understand why there is such a strong female bias for some autoimmune diseases, and hypothesized that there must be a role for X-chromosome inactivation," states corresponding author Montserrat Anguera.

The researchers first investigated patterns of Xist RNA transcripts in mouse $\mathrm{T}$ cells at various stages of differentiation and activation. "We used RNA fluorescence in situ hybridization (FISH) to visualize the localization patterns for the Xist RNA within the nuclei of T cells," explains Anguera. "In resting cells, Xist RNA transcripts were missing from the inactive $\mathrm{X}$, and in stimulated cells Xist RNA coated the inactive X."

In NZB/W F1 female mice, which spontaneously develop lupus-like disease, patterns of Xist RNA transcripts in $\mathrm{CD}^{+} \mathrm{T}$ cells at pre-disease and early disease stages were similar to those in cells from healthy female wild-type mice. However, during late-stage disease, Xist RNA was mislocalized in NZB/W F1 female mice compared with wild-type female mice, being present in $40 \%$ of resting $\mathrm{T}$ cells and occurring in dispersed patterns in stimulated T cells.

Similar changes to Xist RNA patterns were seen in resting and stimulated $\mathrm{T}$ cells from patients with SLE compared with cells from healthy individuals, indicating that part of the X chromosome was reactivated in SLE.

"The most important finding from this work is that there are dispersed Xist RNA transcripts across the nuclei in T cells from patients with SLE, instead of robust localization at the X chromosome," says Anguera. "This observation suggested to us that gene expression from the $\mathrm{X}$ chromosome might be affected, and we discovered that over a hundred X-linked genes were overexpressed in $\mathrm{CD} 4^{+} \mathrm{T}$ cells from patients with SLE."

These X-linked genes included those known to escape $\mathrm{X}$-chromosome inactivation, such as $K D M 5 C$ and $J P X$, and important immuneregulatory genes that would normally be transcriptionally silenced, such as FOXP3 and IL2RG.

"Moving forward, we are curious to investigate whether the regulation of X-chromosome inactivation is also perturbed in B cells," concludes Anguera.

Joanna Collison

ORIGINAL ARTICLE Syrett, C. M. et al. Altered $X$-chromosome inactivation in $\mathrm{T}$ cells may promote sex-biased autoimmune diseases. $\mathrm{Cl}$ Insight $\mathbf{4}$, e126751 (2019) 